日本原子力研究開発機構機関リポジトリ

Japan Atomic Energy Agency Institutional Repository

\begin{tabular}{|c|l|}
\hline Title & $\begin{array}{l}\text { Impact of plasma parameter on self-organization of electron } \\
\text { temperature gradient driven turbulence }\end{array}$ \\
\hline Author(s) & Kawai Chika, Idomura Yasuhiro, Maeyama Shinya, Ogawa Yuichi \\
\hline Citation & Physics of Plasmas, 24(4),p.042303_1-042303_13 \\
\hline Text Version & Publisher's Version \\
\hline URL & $\underline{\text { https://jopss.jaea.go.jp/search/servlet/search?5059247 }}$ \\
\hline DOI & $\underline{\text { https://doi.org/10.1063/1.4980005 }}$ \\
\hline Right & $\begin{array}{l}\text { This article may be downloaded for personal use only. Any other } \\
\text { use requires prior permission of the author and the American } \\
\text { Institute of Physics. } \\
\text { The following article appeared in Physics of Plasmas and may be } \\
\text { found at https://doi.org/10.1063/1.4980005. }\end{array}$ \\
\hline
\end{tabular}




\section{Impact of plasma parameter on self-organization of electron temperature gradient driven turbulence}

C. Kawai, Y. Idomura, S. Maeyama, and Y. Ogawa

Citation: Physics of Plasmas 24, 042303 (2017); doi: 10.1063/1.4980005

View online: http://dx.doi.org/10.1063/1.4980005

View Table of Contents: http://aip.scitation.org/toc/php/24/4

Published by the American Institute of Physics

\section{Articles you may be interested in}

Multi-field/-scale interactions of turbulence with neoclassical tearing mode magnetic islands in the DIII-D tokamak

Physics of Plasmas 24, 056106056106 (2017); 10.1063/1.4977533

Conservation laws for collisional and turbulent transport processes in toroidal plasmas with large mean flows Physics of Plasmas 24, 020701020701 (2017); 10.1063/1.4975075

Electron holes in phase space: What they are and why they matter Physics of Plasmas 24, 055601055601 (2017); 10.1063/1.4976854

Validation of nonlinear gyrokinetic simulations of L- and I-mode plasmas on Alcator C-Mod Physics of Plasmas 24, 056104056104 (2017); 10.1063/1.4977466

Electromagnetic electron temperature gradient driven instability in toroidal plasmas Physics of Plasmas 24, 024501024501 (2017); 10.1063/1.4975189

Symmetry breaking of ion temperature gradient mode structure: From local to global analysis Physics of Plasmas 24, 042502042502 (2017); 10.1063/1.4978947

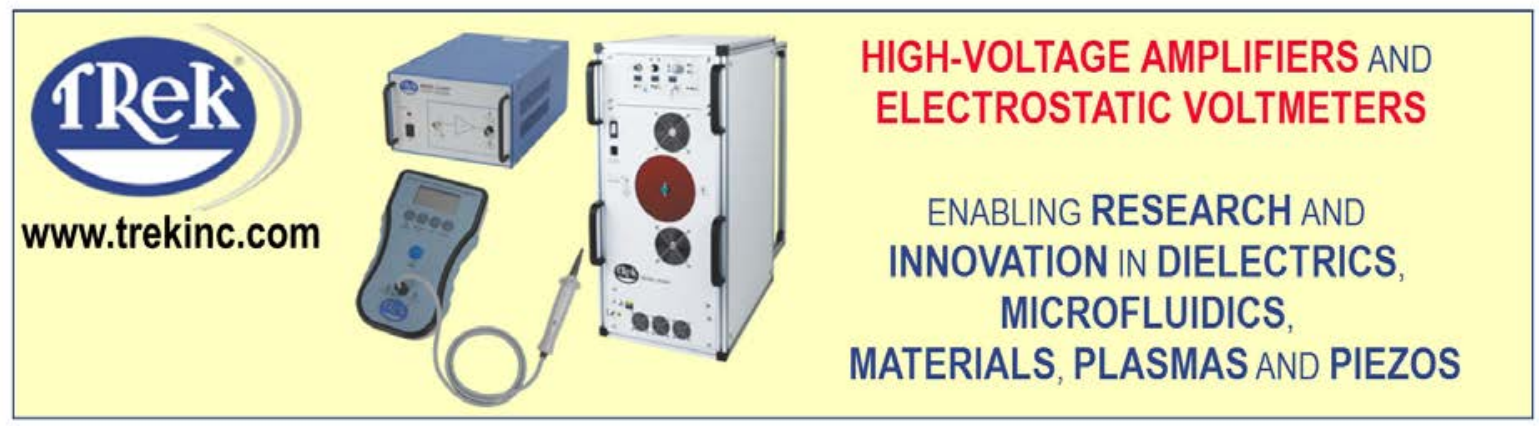




\title{
Impact of plasma parameter on self-organization of electron temperature gradient driven turbulence
}

\author{
C. Kawai, ${ }^{1, a)}$ Y. Idomura, ${ }^{2}$ S. Maeyama, ${ }^{3}$ and Y. Ogawa ${ }^{1}$ \\ ${ }^{1}$ Graduate School of Frontier Science, University of Tokyo, 5-1-5, Kashiwa-no-Ha, Kashiwa, Chiba 277-8568, \\ Japan \\ ${ }_{2}^{2}$ Japan Atomic Energy Agency, 178-4-4, Wakashiba, Kashiwa, Chiba 277-0871, Japan \\ ${ }^{3}$ Department of Physics, Nagoya University, Nagoya 464-8602, Japan
}

(Received 18 October 2016; accepted 27 March 2017; published online 13 April 2017)

\begin{abstract}
Self-organization in the slab electron temperature gradient driven (ETG) turbulence is investigated based on gyrokinetic simulations and the Hasegawa-Mima (HM) equation. The scale and the anisotropy of self-organized turbulent structures vary depending on the Rhines scale and the characteristic scale given by the adiabatic response term in the HM equation. The former is determined by competition between the linear wave dispersion and the nonlinear turbulent cascade, while the latter is given as the scale, at which the turbulent cascade is impeded. These scales are controlled by plasma parameters such as the density and temperature gradient, and the temperature ratio of ion to electron. It is found that depending on the plasma parameters, the ETG turbulence shows either isotropic turbulence or zonal flows, which give significantly different transport levels. Although the modulational instability excites zonal modes regardless of the plasma parameters, the final turbulent structure is determined by the self-organization process. Published by AIP Publishing.

[http://dx.doi.org/10.1063/1.4980005]
\end{abstract}

\section{INTRODUCTION}

Turbulence driven by the inhomogeneity of plasmas such as the density and temperature gradients is considered to be an important mechanism that induces anomalous transport in magnetically confined plasmas. ${ }^{1}$ To achieve improved confinement performance, it is crucial to understand and control plasma turbulence. In this work, we investigate mechanisms of turbulent structure formations or selforganization in the electron temperature gradient driven (ETG) turbulence. Recent multi-scale gyrokinetic (GK) simulations involving both ion and electron scale fluctuations showed that the ETG turbulence might be suppressed when ion scale turbulence is strongly excited. ${ }^{2,3}$ On the contrary, when ion scale turbulence is suppressed by strong $E \times B$ shear flows, electron scale turbulence is dominated by the streamer and thus becomes the main channel of the electron heat losses. ${ }^{4}$ In fact, transport barriers ${ }^{5,6}$ and spherical tokamaks $^{7}$ are characterized by strong $E \times B$ shear flows and ion heat transport at neoclassical levels, and the remaining anomalous electron heat transport is often discussed based on the ETG turbulence.

In positive magnetic shear regions, the toroidal ETG turbulence typically shows radially elongated streamers, which strongly enhance electron heat transport. ${ }^{8,9}$ On the other hand, in weak or zero magnetic shear regions, turbulent structures show slab like features, because toroidal mode coupling becomes weak and turbulent fluctuations are characterized by single helicity or two-dimensional (2D) structures. ${ }^{10}$ The slab ETG turbulence has been studied as a simple model for such a situation, and it was pointed out that electron anomalous heat transport is largely suppressed

${ }^{a)}$ E-mail: c-kawai@ppl.k.u-tokyo.ac.jp when electron scale zonal flows are generated. ${ }^{11,12}$ The suppression of electron heat transport by the electron scale zonal flows is also confirmed in the toroidal ETG turbulence. ${ }^{13}$

The generation and saturation mechanisms of ETG zonal flows have been discussed based on various secondary and tertiary instabilities such as the modulational instability driven by pump waves, ${ }^{14,15}$ and the Kelvin-Helmholtz-like instabilities excited by shear flows in linear streamers ${ }^{8,16}$ and in zonal flows. ${ }^{11}$ On the other hand, another mechanism to determine ETG zonal flows was discussed based on the selforganization in 2D rotating fluid turbulence. ${ }^{17}$ Here, the selforganization denotes turbulent structure formations not at the box size, but at system specific scales such as the Rhines scale and the adiabatic scale. The quasi-2D slab ETG turbulence is well described by the Hasegawa-Mima (HM) equation, ${ }^{18}$ which is mathematically equivalent to the Charney equation describing the Rossby wave turbulence. ${ }^{19}$ In such $2 \mathrm{D}$ rotating fluid turbulence, short wavelength turbulence is characterized by the so-called dual cascade with the inverse energy cascade.

In gyrokinetic simulations, the turbulent cascade and the resulting turbulent spectra in the sub-Larmor scale range are often discussed based on the so-called entropy cascade ${ }^{20}$ induced by phase mixing due to perpendicular $E \times B$ drifts with the finite Larmor radius (FLR) effect. ${ }^{21,22}$ However, the above classical self-organization process described by the HM equation and the resulting turbulent spectra in the long wavelength range have not been directly observed in gyrokinetic simulations, while the correspondence between the Rhines scale and the scale of ETG zonal flows was shown in gyrokinetic particle simulations. ${ }^{17}$

In the self-organization picture, two competing effects, the inverse energy cascade and the linear wave dispersion, 
are characterized by the nonlinear transfer rate or the inverse of the eddy turnover time $\omega_{t}$, and the linear wave frequency $\omega_{r}$. If the self-organization occurs at the Rhines scale or the wave-turbulence boundary $\omega_{t} \sim \omega_{r}$, the formation of zonal flows is expected as in the Rossby wave turbulence. However, this picture may be modified in the ETG turbulence, where the adiabatic response term is not negligible. When its characteristic wave number $k_{\lambda}$ is much higher than that for the Rhines scale $k_{c}$, the turbulent inverse energy cascade is impeded by the adiabatic response term with keeping the isotropic turbulent structure. This means that the turbulent structures may be controlled by changing either $k_{\lambda}$ or $k_{c}$, which depends on various plasma parameters, e.g., density and temperature gradient, and temperature ratio. In this study, we examine the self-organization process in the slab ETG turbulence through direct measurements of turbulent spectra using the gyrokinetic Eulerian code G5D. ${ }^{23}$ The comparisons of gyrokinetic simulations against fluid simulations based on the HM equation and the ETG turbulence simulations give the viability of self-organization picture in the gyrokinetic (GK) turbulence, while the comparison of ETG turbulence simulations with different plasma parameters shows its effect on the structure formation of ETG turbulence.

The remainder of this article is organized as follows. In Sec. II, a GK model used in this study is introduced, and its relation to the HM equation is discussed. In Sec. III, decaying turbulence simulations with the GK and the HM models are presented, and basic properties of the selforganization process are discussed. In Sec. IV, ETG turbulence simulations are shown, and the influences of plasma parameters on the turbulent structures and the mechanism of self-organization are discussed. Finally, Sec. V gives the summary and discussion.

\section{CALCULATION MODEL}

\section{A. Gyrokinetic model}

The electrostatic gyrokinetic equations for the electron distribution function $F\left(\boldsymbol{R}, v_{\|}, \mu ; t\right)$ in a slab configuration are given by ${ }^{17}$

$$
\begin{aligned}
\frac{\partial F}{\partial t}+ & \left(v_{\|} \boldsymbol{b}+\frac{c}{B_{0}} \boldsymbol{b} \times \nabla\langle\phi\rangle_{\alpha}\right) \cdot \nabla F \\
& -\frac{q_{e}}{m_{e}} \boldsymbol{b} \cdot \nabla\langle\phi\rangle_{\alpha} \frac{\partial F}{\partial v_{\|}}=C(F), \\
& -\left(\nabla^{2}+\frac{\rho_{T e}^{2}}{\lambda_{D e}^{2}} \nabla_{\perp}^{2}\right) \phi+\frac{1}{\lambda_{D i}^{2}} \phi \\
= & 4 \pi q_{e}\left[\int F \delta[(\boldsymbol{R}+\boldsymbol{\rho})-\boldsymbol{x}] \mathrm{d}^{6} Z-n_{0}\right],
\end{aligned}
$$

where $\boldsymbol{R}$ is the guiding center position, $\boldsymbol{R}+\boldsymbol{\rho}$ is the particle position, $v_{\|}$is the parallel velocity, $\mu$ is the magnetic moment, $\alpha$ is the gyro-phase angle, $\phi$ is the electrostatic potential, $m_{s}$ and $q_{s}$ are the mass and charge of the $s$ th species, $\boldsymbol{b} \equiv \boldsymbol{B} /|\boldsymbol{B}|$ is a unit vector in the direction of the magnetic field $\boldsymbol{B}=B_{0} \hat{\boldsymbol{z}}+B_{1} \hat{\boldsymbol{y}}, \lambda_{D s} \equiv \sqrt{T_{s} / 4 \pi n_{s} q_{s}^{2}}$ is the Debye length, $\rho_{T e} \equiv v_{T e} / \Omega_{e}$ is the electron Larmor radius,
$v_{T e} \equiv \sqrt{T_{e} / m_{e}}$ is the electron thermal velocity, $\Omega_{e} \equiv q_{e} B_{0} /$ $\left(m_{e} c\right)$ is the electron cyclotron frequency, $n_{0}(x)$ is the background ion density, $\langle\cdot\rangle_{\alpha} \equiv \frac{1}{2 \pi} \int \cdot \mathrm{d} \alpha$ is the gyro-average operator, and $C(F)$ is a linear Fokker-Planck collision operator. $^{24,25}$ In Eq. (2), the adiabatic ion response $n_{i}=n_{0}$ $-n_{0} q_{i} \phi / T_{i}$ and the long wavelength approximation, $k^{2} \rho_{T e}^{2}$ $\ll 1$, are assumed.

Eqs. (1) and (2) are solved using the full- $f$ gyrokinetic Eulerian code G5D. ${ }^{23}$ In a shearless slab configuration, single helicity perturbations with $k_{\|}=k_{y} B_{1} /|\boldsymbol{B}|$ are solved with a fixed boundary condition, e.g., $\phi=0$ and $F=0$ at the boundary, in the $x$-direction and a periodic condition in the $y$-direction. The initial equilibrium distribution function $F_{0}$ is given by a local Maxwellian distribution with the following density and temperature profiles:

$$
\begin{aligned}
n(x) & =\bar{n} \exp \left[-\frac{0.3 L_{x}}{L_{n}} \tanh \left(\frac{x-0.5 L_{x}}{0.3 L_{x}}\right)\right], \\
T_{e}(x) & =\bar{T}_{e} \exp \left[-\frac{0.3 L_{x}}{L_{T e}} \tanh \left(\frac{x-0.5 L_{x}}{0.3 L_{x}}\right)\right],
\end{aligned}
$$

where $\bar{n}$ and $\bar{T}_{e}$ are the density and the temperature at $x=0.5 L_{x}$, and $L_{n} \equiv n /|\nabla n|$ and $L_{T e} \equiv T_{e} /\left|\nabla T_{e}\right|$ are the scale lengths of density and temperature gradients, respectively. The box size in the $x$ and $y$ directions is given as $\left(L_{x}, L_{y}\right)=\left(585 \rho_{T e}, 292 \rho_{T e}\right)$, and the grid number is chosen as $N_{x} \times N_{y} \times N_{v_{\|}} \times N_{\mu}=512 \times 256 \times 64 \times 16$, such that turbulent energy injection and dissipation are correctly computed by covering linearly unstable and stable wavenumber ranges.

\section{B. Hasegawa-Mima model}

By neglecting the collision term, the parallel dynamics ( $k_{\|} \rightarrow 0$ or 2D limit), and the gyro-average operator, Eqs. (1) and (2) yield the HM equation for electron scale turbulence

$$
\frac{\partial}{\partial t}\left(\rho_{s}^{2} \nabla_{\perp}^{2} \phi-\tau \phi\right)+(\boldsymbol{b} \times \nabla \phi) \cdot \nabla\left(\rho_{s}^{2} \nabla^{2} \phi+\ln n_{0}\right)=0,
$$

where $\tau \equiv T_{e} / T_{i}$, and $\rho_{s}^{2} \equiv 1+\lambda_{D e}^{2} / \rho_{T e}^{2}$ involves a correction due to the Debye shielding term. In Eq. (5), normalizations are chosen as $\hat{x} \equiv x / \rho_{T e}, \hat{t} \equiv t\left|\Omega_{e}\right|, \hat{v} \equiv v / v_{T e}, \hat{n} \equiv n / n_{0}$ by using the density and the temperature at the reference surface or $x=0.5 L_{x}$, and hereafter, the hat is suppressed for simplicity. The term $\tau \phi$ in Eq. (5) originates from the second term in Eq. (2), which corresponds to the ion adiabatic response term. By linearising Eq. (5), the linear dispersion of the electron drift wave is obtained as

$$
\omega_{r}=\frac{L_{n}^{-1} k_{y}}{\tau+k^{2} \rho_{s}^{2}},
$$

where $k^{2}=k_{x}^{2}+k_{y}^{2}$.

The HM equation conserves two quantities, the energy $E=\frac{1}{V} \int \frac{1}{2}\left[\tau|\phi|^{2}+\rho_{s}^{2}|\nabla \phi|^{2}\right] \mathrm{d} V$, and the enstrophy $W=\frac{1}{V} \int \frac{1}{2}$ $\left[\tau|\nabla \phi|^{2}+\rho_{s}^{2}\left|\nabla^{2} \phi\right|^{2}\right] \mathrm{d} V$, where $\frac{1}{V} \int \cdot d V$ denotes the volume average. The conservation of these two quantities induces 
the dual cascade, the inverse energy cascade, and the normal enstrophy cascade, which imposes different power laws for the turbulent energy spectra $E_{k}$ in the long and short wavelength ranges 26

$$
E_{k} \propto \begin{cases}k^{-5 / 3} & \left(k<k_{s}\right), \\ k^{-3} & \left(k>k_{s}\right),\end{cases}
$$

where $k_{s}$ is the energy injection scale at which turbulence is driven.

However, in rotating fluids such as drift wave turbulence, the inverse energy cascade is impeded at a specific scale because of a frequency mismatch induced by the linear wave dispersion originated from the fluid rotation. The rate at which energy cascade evolves due to the nonlinear coupling is estimated by the inverse of the eddy turnover time $\omega_{t}{ }^{27}$ It is noted that in Ref. 27, the nonlinear transfer rate is defined as

$$
\omega_{t}=k U,
$$

assuming $k^{2} \rho_{s}^{2} \gg 1$. Here, $U=(2 \epsilon)^{1 / 2}$ is the RMS turbulent velocity, $\epsilon \equiv \frac{1}{V} \int \frac{1}{2}|\nabla \phi|^{2} \mathrm{~d} V$, and $\theta=\tan ^{-1}\left(k_{y} / k_{x}\right)$. Instead of using this definition, we measure the nonlinear transfer rate at an arbitrary scale based on the nonlinear term of the HM equation retaining the adiabatic response term

$$
\hat{\omega}_{t}=\frac{k^{3} \rho_{s}^{2}}{\tau+k^{2} \rho_{s}^{2}} U
$$

Equating the linear drift wave frequency $\omega_{r}$ and the nonlinear transfer rate $\hat{\omega}_{t}$ yields a critical wavenumber $k_{c, x}$ and $k_{c, y}{ }^{27}$

$$
\begin{aligned}
& k_{c, x} \rho_{s}=L_{n}^{-1 / 2} U^{-1 / 2} \sin ^{1 / 2} \theta \cos \theta, \\
& k_{c, y} \rho_{s}=L_{n}^{-1 / 2} U^{-1 / 2} \sin ^{3 / 2} \theta,
\end{aligned}
$$

and its isotropic estimation by setting $\theta=\pi / 2$

$$
k_{c} \rho_{s}=L_{n}^{-1 / 2} U^{-1 / 2} .
$$

The linear wave dispersion becomes important in the low $k$ range $k<k_{c}$, while the nonlinear turbulent cascade is dominant in the high $k$ range $k>k_{c}$. Therefore, $k_{c}$ gives the Rhines scale, ${ }^{28}$ at which a spectral condensation is expected.

There is another scale that divides the property of turbulent inverse energy cascade. According to Eq. (5), the adiabatic response term $\tau \phi$ becomes comparable to the Debye shielding term $\rho_{s}^{2} \nabla^{2} \phi$ at the adiabatic response scale $k_{\lambda}$

$$
k_{\lambda} \rho_{s}=\tau^{1 / 2} .
$$

It is thus expected that while Eq. (5) has almost the same property as the 2D Navier-Stokes equation in the higher $k$ range $k \gg k_{\lambda}$, the dynamics of the turbulence in the lower $k$ range $k \ll k_{\lambda}$ is affected by the adiabatic response term. This leads to different power laws for $E_{k}$ across $k_{\lambda}{ }^{29}$

$$
E_{k} \propto \begin{cases}k^{-11 / 3} & \left(k \ll k_{\lambda}\right), \\ k^{-5 / 3} & \left(k_{\lambda} \ll k \ll k_{s}\right)\end{cases}
$$

This also gives different time evolutions of turbulent spectra. In Ref. 29, the characteristic wavenumber $k_{E}$ for the energy containing scale is defined as

$$
k_{E} \equiv \frac{\int \mathrm{d} k_{x} \mathrm{~d} k_{y}|k| E_{k_{x}, k_{y}}}{\int \mathrm{d} k_{x} \mathrm{~d} k_{y} E_{k_{x}, k_{y}}},
$$

and its time dependency $k_{E}(t)$ is estimated by dimensional analysis as

$$
k_{E}(t) \sim \begin{cases}t^{-3 / 8} & \left(k_{E} \ll k_{\lambda}\right), \\ t^{-3 / 2} & \left(k_{E} \gg k_{\lambda}\right) .\end{cases}
$$

The difference of power laws indicates that once energy containing scale reaches around $k_{\lambda}$ by the inverse energy cascade, the process slows down and the energy spectrum is expected to condensate around this scale.

The formation of zonal flows is expected when the energy spectrum stagnates around $k_{c}$ due to the linear dispersion. On the contrary, when the slowing down of the inverse energy cascade around $k_{\lambda}$ occurs around higher $k$ range than $k_{c}$, the formation of an isotropic turbulence structure is anticipated since the contribution from the linear term is smaller than that from the nonlinear term around the energy containing scale $k_{E}$. Therefore, the relative magnitude of $k_{c}$ and $k_{\lambda}$, which are dependent on $L_{n}$ and $U$, and $\tau$, respectively, is of critical importance in dictating turbulence structures. The scale lengths discussed so far are summarized in Table I.

Nonlinear simulations of the HM model are performed using a pseudo-spectral method with including the hyperviscosity term

$$
\begin{aligned}
& -\frac{\partial}{\partial t}\left(\tau+k^{2} \rho_{s}^{2}\right) \phi_{k}+\frac{1}{2} \sum_{k=k^{\prime}+k^{\prime \prime}} \\
& \quad \times\left[\boldsymbol{b} \cdot\left(k^{\prime} \times k^{\prime \prime}\right)\left(k^{\prime \prime 2}-k^{\prime 2}\right) \rho_{s}^{2} \phi_{k^{\prime}} \phi_{k^{\prime \prime}}\right]+i L_{n}^{-1} k_{y} \phi_{k}=\mu k^{4} \phi_{k},
\end{aligned}
$$

where the dissipation coefficient is chosen as $\mu=1.0 \times 10^{-3}$. Periodic boundary conditions are imposed in both the $x$ - and $y$-directions by assuming a local approximation with constant $L_{n}$. The simulation parameters and the grid number are chosen to be the same as the GK model, while the HM model is considered in the $2 \mathrm{D}$ limit or $B_{1} \rightarrow 0$.

\section{Linear wave dispersion in the HM and the GK models}

The Rhines scale is determined by the two competing effects, the linear wave dispersion and the turbulent cascade,

TABLE I. The summary of characteristic scales.

\begin{tabular}{lc}
\hline \hline Rhines scale & $k_{c}=\rho_{s}^{-1} L_{n}^{-1 / 2} U^{-1 / 2}$ \\
Adiabatic response scale & $k_{\lambda}=\rho_{s}^{-1} \tau^{1 / 2}$ \\
Scale length of zonal flows & $k_{Z F}=\frac{\int \mathrm{d} k_{x} \mathrm{~d} k_{y} k_{x} E_{k_{x}, k_{y}=0}}{\int \mathrm{d} k_{x} \mathrm{~d} k_{y} E_{k_{x}, k_{y}=0}}$ \\
Energy containing scale & $k_{E}=\frac{\int \mathrm{d} k_{x} \mathrm{~d} k_{y}|k| E_{k_{x}, k_{y}}}{\int \mathrm{d} k_{x} \mathrm{~d} k_{y} E_{k_{x}, k_{y}}}$ \\
Energy injection scale & $k_{s}$ \\
\hline \hline
\end{tabular}



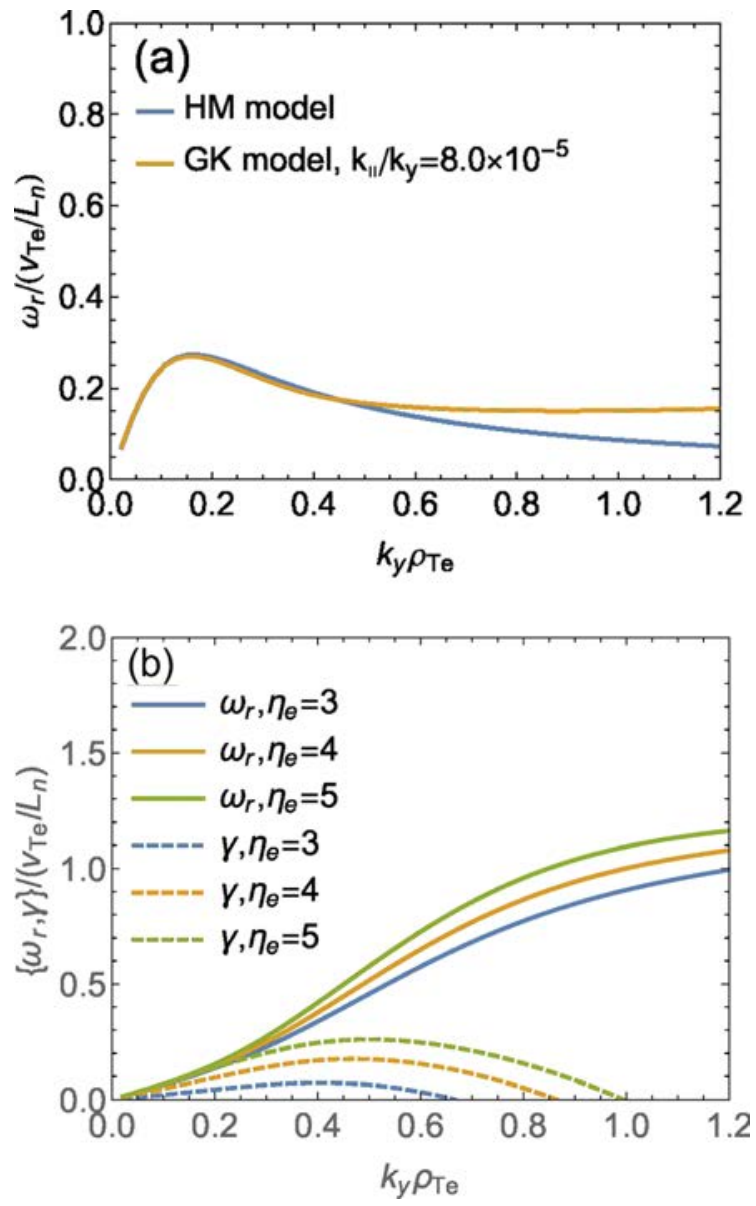

FIG. 1. In (a), the real frequency of the linearly stable electron drift wave in the decaying turbulence with $\eta_{e}=0, \tau=0.3$, and $\rho_{s}^{2}=11$ is computed using the HM model $\left(k_{\|}=0\right)$ and the GK models $\left(k_{\|} / k_{y}=8.0 \times 10^{-5}\right)$. In (b), the $\eta_{e}$ dependency of the real frequency and the growth rate of the ETG mode are calculated using the GK model. Here, $\eta_{e}$ is changed from the reference parameters with $\tau=0.3, \rho_{s}^{2}=2$, and $k_{\|} / k_{y}=5.1 \times 10^{-4}$.

which are characterized by the linear frequency $\omega_{r}$ and the nonlinear transfer rate $\omega_{t}$, respectively. Therefore, it is important to estimate the linear wave dispersion in the HM and GK models. In this section, we estimate linear dispersion relations by solving an eigenvalue problem ${ }^{30}$ in the local limit, where radial variations of $L_{n}$ and $L_{T e}$ are neglected, and a periodic radial boundary condition is assumed.

Figure 1(a) shows a comparison of $\omega_{r}$ of the linearly stable electron drift wave in the decaying turbulence simulations in Sec. III. In the high $k_{y}$ range, the spectra obtained from the GK and the HM models show some difference due to the FLR effect, while in the low $k_{y}$ range, they show a quantitative agreement. Figure 1(b) shows the $\eta_{e} \equiv L_{n} / L_{T e}$ dependency of $\omega_{r}$ and the growth rate $\gamma$ of the ETG mode, which is discussed in Sec. IV. In the $\eta_{e}$ scan, $\gamma$ is largely varied, while the change in $\omega_{r}$ is relatively small.

In Figs. 2(a)-2(c), the ratios of $\omega_{r}$ to $\hat{\omega}_{t}$ in the decaying turbulence simulations are plotted on the $k_{x}-k_{y}$ plane. Here, $\hat{\omega}_{t}$ is estimated by using the RMS turbulent velocity $U$ in the decaying turbulence simulations in Figs. 4(b)-4(d). In the figures, the thick lines show the 2D structures of the Rhines scale $k_{c}$ at which $\omega_{r}=\hat{\omega}_{t}$. In both HM and GK models, $k_{c}$ shows similar shapes, in which the
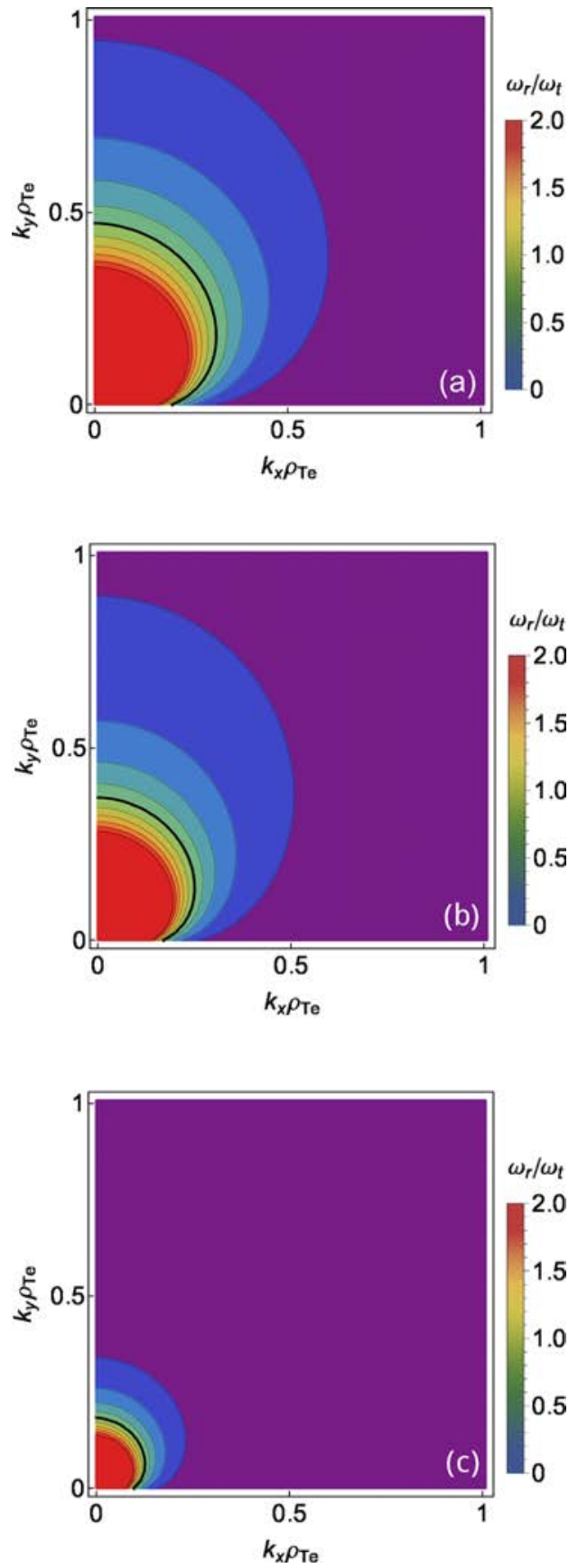

FIG. 2. The ratio of the real frequency $\omega_{r}$ to the nonlinear transfer rate $\hat{\omega}_{t}$ is plotted on the $k_{x}-k_{y}$ plane. $\omega_{r}$ and $\hat{\omega}_{t}$ are estimated for the decaying turbulence simulations with (a) weak turbulence case $\left(U \sim 2.55 \times 10^{-4}\right)$ in the HM model, (b) weak turbulence case $\left(U \sim 3.62 \times 10^{-4}\right)$ in the GK model, and (c) strong turbulence case $\left(U \sim 2.15 \times 10^{-3}\right)$ in the GK model. The thick line shows the Rhines scale $k_{c}$ at which $\omega_{r}=\hat{\omega}_{t}$.

inverse energy cascade is impeded in the wave-like region with $k_{y}>k_{x}$, and a spectral condensation at $k_{y} \sim 0$ is expected. In the weak turbulence case (see Fig. 2(b)), $k_{c}$ is rather close to the initial perturbation with $k \rho_{T e} \sim 0.6$, while in the strong turbulence case (see Fig. 2(c)), the wave-like region shrinks to the lower $k$ region. The structure of strong turbulence might thus be different from that of weak turbulence. These conjectures are examined in the decaying turbulence simulations in Sec. III. 


\section{DECAYING TURBULENCE}

In this section, decaying turbulence simulations are performed based on the HM and GK models, and their selforganization processes are investigated. In the decaying turbulence simulations, isotropic and random density perturbations are initially given around $k \rho_{T e} \sim 0.6$ (see Fig. 4(a)). Since the initial perturbations do not involve coherent pump waves, the modulational instability is not expected in the decaying turbulence, and one can focus on the selforganization processes. The initial perturbations are damped either by the hyperviscosity in the HM model or by the Landau damping in the GK model, and self-organized turbulent structures are formed after the turbulence relaxation processes. The reference parameters for the decaying turbulence simulations are chosen as $\tau=0.3, \rho_{s}^{2}=11, \eta_{e}=0$.

\section{A. Turbulent energy spectra in the HM and the GK models}

In this section, $k_{\|} / k_{y}=2.7 \times 10^{-4}$ is chosen so that the damping of the GK model is comparable to that in the HM model. Figure 3 shows the time development of the RMS turbulence velocity $U$ for the HM and GK models with weak perturbations and the GK model with strong perturbation. In the following, physical quantities in decaying turbulence simulations are measured at the quasi-steady phase with time averaging over $\Delta t=40 L_{n} / v_{T e}$. In Figs. 4(b) and 4(c), the 2D turbulent energy spectra

$$
E_{k_{x}, k_{y}} \equiv\left(\tau+k^{2} \rho_{s}^{2}\right)\left|\phi_{k_{x}, k_{y}}\right|^{2},
$$

are shown for the weak turbulence cases in which the maximum initial perturbation amplitude is given as $\left|q_{e} \phi / T_{e}\right|=0.5 \%$. Although the final turbulence amplitudes differ because of different dissipation mechanisms, both the HM and GK models generate the similar self-organized turbulent structures with zonal flows. In the spectra, the spectral condensation occurs outside the wave-like region in Figs. 2(b) and 2(c), and the anisotropic turbulent structures with the so-called dumb-bell shape are formed. These observations indicate that the self-organization picture of the HM model is relevant also in the GK model.

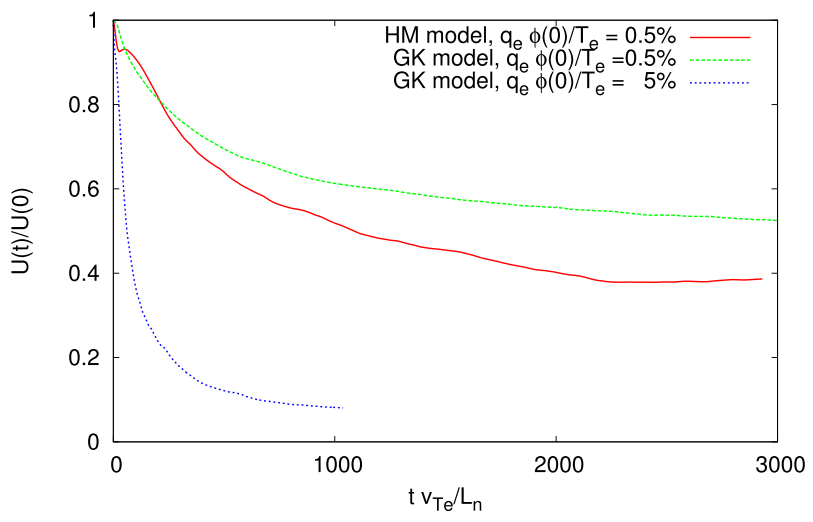

FIG. 3. Time histories of the RMS turbulence velocity $U$ for the decaying turbulence simulations of HM and GK models with $q \phi(0) / T_{e}=0.5 \%, 5 \%$.

\section{B. Density gradient scan}

In this section, the $L_{n}$ dependency of zonal flows is studied to verify the present decaying turbulence simulations against the Rhines scale and the former particle simulations. ${ }^{17}$ The $L_{n}$ scan of decaying turbulence is performed using the GK model with $k_{\|}=8.0 \times 10^{-5}$ following Ref. 17, and the characteristic scale of zonal flows:

$$
k_{Z F} \equiv \frac{\int \mathrm{d} k_{x} \mathrm{~d} k_{y} k_{x} E_{k_{x}, k_{y}=0}}{\int \mathrm{d} k_{x} \mathrm{~d} k_{y} E_{k_{x}, k_{y}=0}},
$$

is compared with the Rhines scale $k_{c}$. In Fig. 5, the $L_{n}$ dependency of $k_{Z F}$ agrees well with that of $k_{c}$, and the former particle simulation results are successfully recovered also in the present Eulerian simulations.

\section{Turbulence amplitude scan}

Another important control parameter is turbulence intensity, which can be changed by the initial perturbation amplitude in the decaying turbulence simulation. As deduced from Eqs. (10) and (11), the turbulence intensity or the RMS turbulent velocity $U$ dictates the Rhines scale. In order to see this effect, the decaying turbulence simulation using the GK model is repeated by imposing larger initial perturbations with $\left|q_{e} \phi / T_{e}\right|=5.0 \%$. In Fig. 4(d), the 2D turbulent energy spectrum clearly shows that both the scale and the anisotropy of the self-organized turbulent structure are significantly changed from the weak turbulence case in Fig. 4(c). This change is consistent with the shrinkage of $k_{c}$ shown in Fig. 2(c).

In order to discuss the anisotropy of turbulent structures quantitatively, we introduce a measure of the anisotropy $c_{y}$, which is defined by the relative magnitude of the turbulent energy to the $E \times B$ drifts in the $y$-direction

$$
c_{y} \equiv \frac{\int \mathrm{d} x \mathrm{~d} y\left|\frac{c}{B_{0}} \hat{\boldsymbol{y}} \cdot(\boldsymbol{b} \times \nabla \phi)\right|^{2}}{\int \mathrm{d} x \mathrm{~d} y\left|\frac{c}{B_{0}}(\boldsymbol{b} \times \nabla \phi)\right|^{2}} .
$$

In the anisotropic limit, zonal flows with $k_{y}=0$ and streamers with $k_{x}=0$ give $c_{y}=1$ and $c_{y}=0$, respectively, while isotropic turbulence gives $c_{y}=0.5$. Table II summarizes $U$, $k_{Z F}$, and $c_{y}$ for the decaying turbulence simulations in Figs. 4(b)-4(d). In the data, it is clearly seen that the selforganized structures characterized by $k_{Z F}$ and $c_{y}$ are qualitatively different between the weak and strong turbulence cases.

It is not expected from Eq. (10) that not only the scale but the anisotropy of the turbulence also depends on the turbulence amplitude. The difference may arise because the adiabatic response term for the electron gyrokinetic turbulence is not negligible, while the short wavelength approximation $\left(\tau+k^{2}\right) \phi \sim k^{2} \phi$ is assumed in the Rossby wave turbulence. In order to see how the dynamics of inverse 

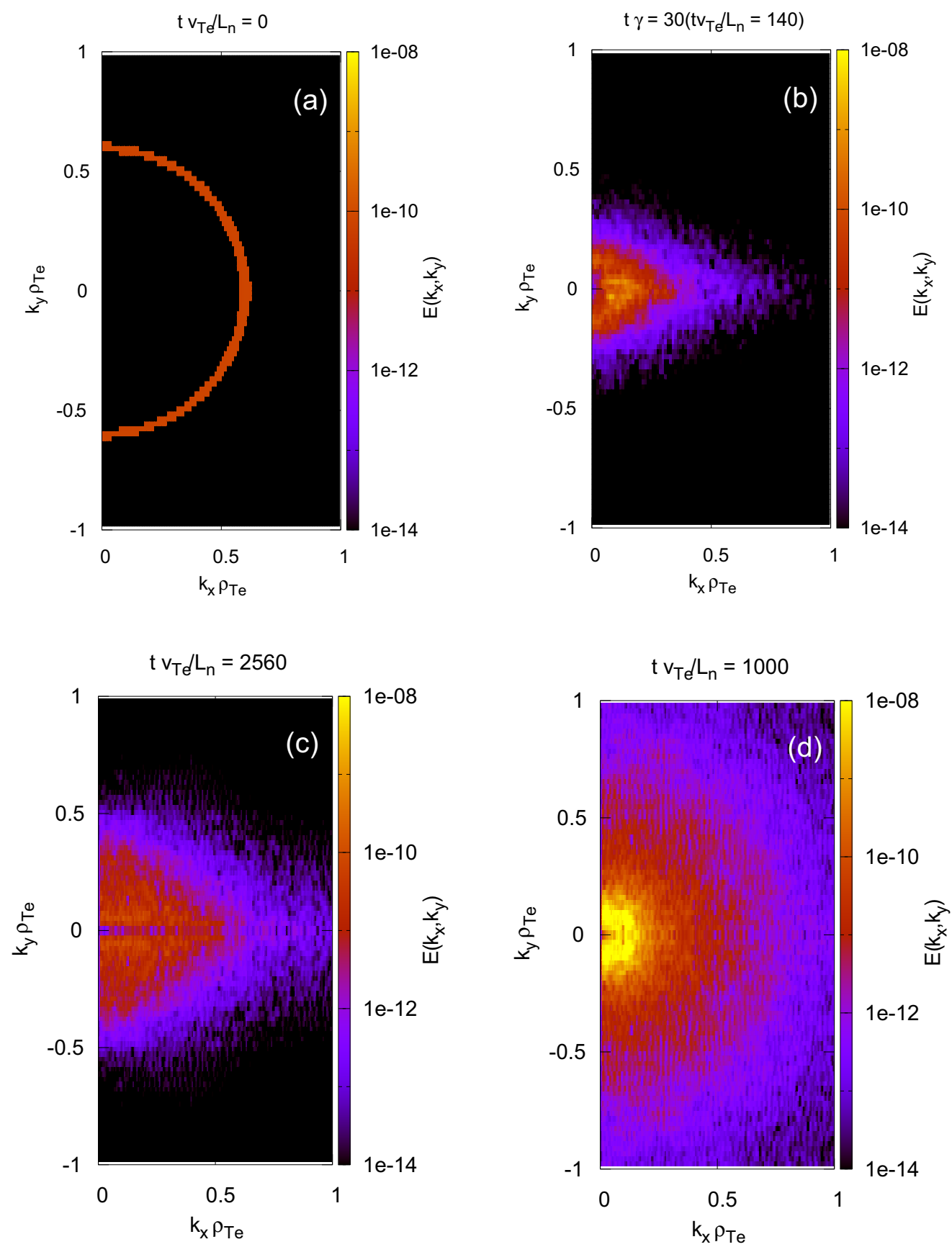

FIG. 4. The $2 \mathrm{D}$ energy spectra $E_{k_{x}, k_{y}}$ in the decaying turbulence simulations are plotted for (a) the initial perturbations and the self-organized turbulent structures of (b) weak turbulence case in the HM model, (c) weak turbulence case in the GK model, and (d) strong turbulence case in the GK model. (b)-(d) Are observed by taking time average for $\Delta t \sim 40 L_{n} / v_{T e}$ in the quasi-steady phases. In the weak and strong turbulence cases, the initial perturbations are given by isotropic and random density perturbations with $\left|q_{e} \phi(t=0) / T_{e}\right| \sim 0.5 \%$ and $\left|q_{e} \phi(t=0) / T_{e}\right| \sim 5 \%$, respectively.

energy cascade affects the turbulent structure, the time development of the energy containing scale $k_{E}(t)$ given by Eq. (14) is plotted for both simulations in Fig. 6. In the strong turbulence case, $k_{E}$ gradually shifts to the lower $k$ following $k_{E}(t) \sim t^{-3 / 8}$. This time dependency suggests that the inverse energy cascade is not blocked by the linear wave dispersion but by the adiabatic response term. Since the linear wave dispersion is weaker than the nonlinear inverse energy cascade at $k_{E}$, the isotropic turbulence is formed. This time dependence is not observed in the weak turbulence case. The energy containing scale for this case is determined by the Rhines scale as shown in Fig. 5, but $k_{E}$ becomes smaller as the short wavelength spectra decay by the Landau damping. The above results indicate that the competition of two mechanisms, which impede the inverse energy cascade, dictates the turbulent structure. One is the linear wave dispersion, which dictates the Rhines scale $k_{c}$ and the other is the adiabatic response term, which characterizes the adiabatic response scale $k_{\lambda}$. The former generates zonal flows, while the latter keeps isotropic turbulence. Therefore, the anisotropy of the turbulent structure is characterized by the ratio $k_{c} / k_{\lambda}$ 


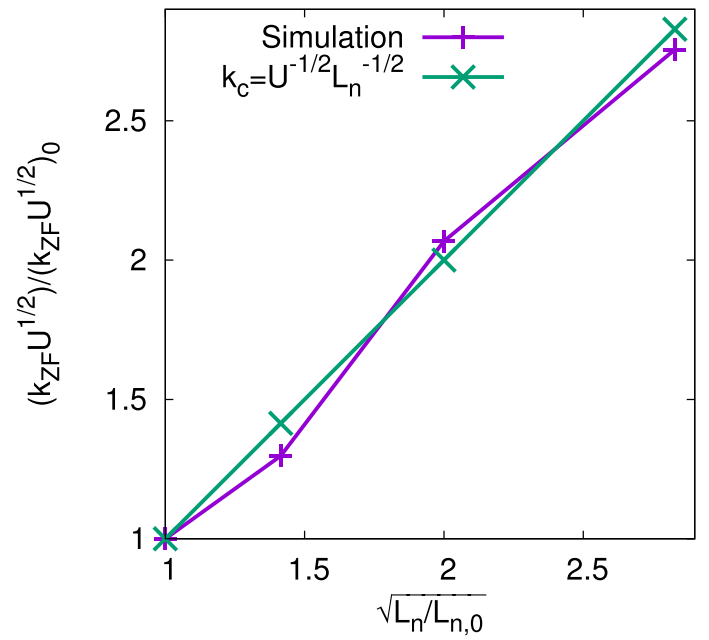

FIG. 5. The $L_{n}$ dependencies of $k_{c}$ and $k_{Z F}$ are compared in the $L_{n}$ scan of the decaying turbulence simulations.

TABLE II. The RMS turbulent velocity $U$, the wavenumber of zonal flows $k_{Z F}$, and the turbulent anisotropy $c_{y}$ for the decaying turbulence simulations in Figs. 4(b)-4(d).

\begin{tabular}{lcccc}
\hline \hline Model & $q_{e} \phi / T_{e}(t=0)$ & $U$ & $k_{Z F} \rho_{T e}$ & $c_{y}$ \\
\hline HM & $0.5 \%$ & $2.55 \times 10^{-4}$ & 0.20 & 0.85 \\
GK & $0.5 \%$ & $3.62 \times 10^{-4}$ & 0.42 & 0.73 \\
GK & $5 \%$ & $2.55 \times 10^{-3}$ & 0.12 & 0.53 \\
\hline \hline
\end{tabular}

$$
\frac{k_{c}}{k_{\lambda}}=L_{n}^{-1 / 2} U^{-1 / 2} \tau^{-1 / 2}
$$

The weak and strong turbulence simulations in Figs. 4(c) and 4(d), respectively, show $k_{c} / k_{\lambda} \sim 2.4$ and $0.97 . k_{c} / k_{\lambda}>1$ indicates that the inverse energy cascade is impeded by the linear wave dispersion, which results in the formation of the anisotropic structure as in the Rhines scale picture. In the opposite case, $k_{c} / k_{\lambda}<1$ implies that inverse energy cascade cannot continue up to the Rhines scale, but is hindered at the adiabatic response scale.

\section{ETG TURBULENCE SIMULATION}

In Sec. III, the decaying turbulence simulations show that the self-organization picture of the HM model is applicable to the GK model. The resulting turbulent structures are controlled by relative intensity between the linear wave dispersion and the turbulent cascade, which are characterized by $\omega_{r}$ and $\hat{\omega}_{t}$ at the energy containing scale $k_{E}$. In this section, we examine these properties in the ETG turbulence simulations, in which quasi-steady turbulent spectra are sustained by a balance between energy injection by the ETG mode and energy dissipation by the Landau damping. In the ETG turbulence simulations, the reference parameters are chosen as $\tau=0.3, \rho_{s}^{2}=2.0$, and $k_{\|} / k_{y}=5.1 \times 10^{-4}$, so that the scales of the energy injection and the energy dissipation are well separated and covered by the present numerical parameters.

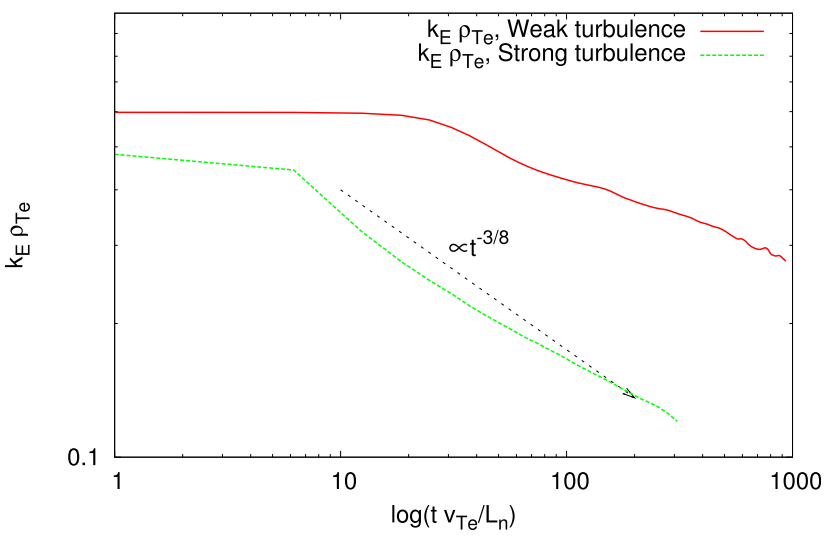

FIG. 6. The time development of the energy containing scale $k_{E}(t)$ is shown for the weak and strong decaying turbulence simulations (see Fig. 4). The inverse energy cascade for $k<k_{\lambda}$ is characterized by $k_{E}(t) \propto t^{-3 / 8}$.

\section{A. Self-organization in ETG turbulence}

Figures 7(a) and 7(b) show the turbulent electrostatic potential in the weak ETG turbulence with $\eta_{e}=2.85$ and in the strong ETG turbulence with $\eta_{e}=5.0$, which are characterized by qualitatively different turbulent structures with zonal flows and with isotropic turbulence, respectively. Reflecting these turbulent structures, the electron heat diffusivity is also changed. The electron heat transport coefficient is calculated by the time and volume average of the local transport coefficient within turbulent regions

$$
\chi_{e}(t)=\frac{1}{0.4 L_{x} L_{y} \Delta t} \int_{t}^{t+\Delta t} \mathrm{~d} t \int_{0}^{L_{y}} \mathrm{~d} y \int_{0.3 L_{x}}^{0.7 L_{x}} \mathrm{~d} x \frac{Q_{e}}{p_{0}^{\prime}},
$$
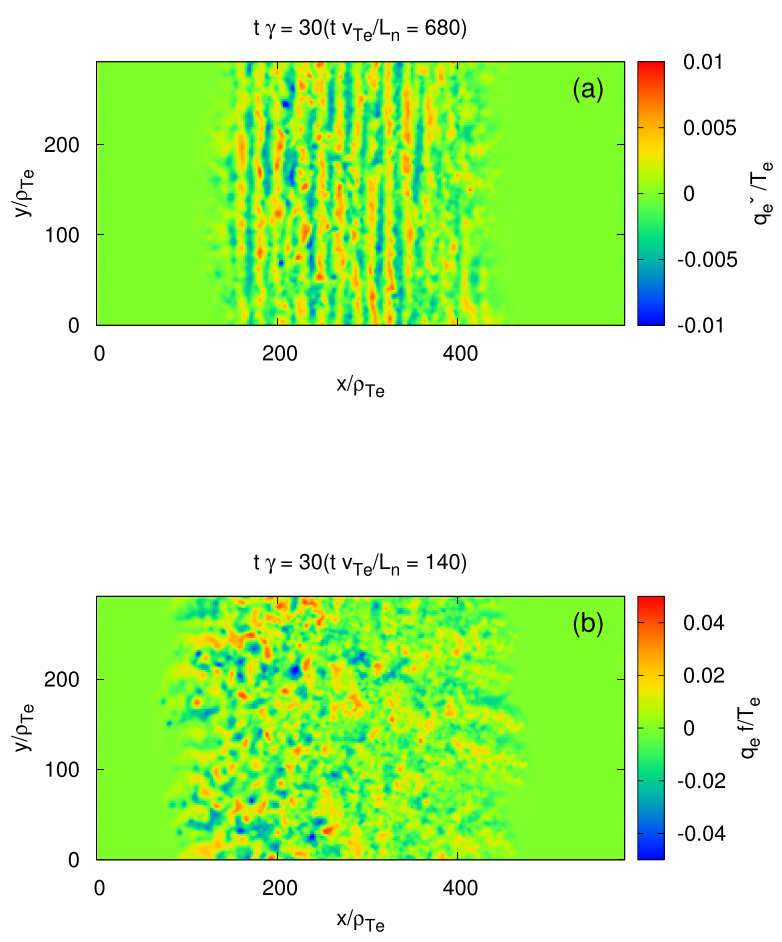

FIG. 7. The electrostatic potential $q_{e} \phi / T_{e}$ in the ETG turbulence is plotted on the $x$ - $y$ plane. (a) The weak ETG turbulence with $\eta_{e}=2.85, \gamma \sim 0.043 v_{T e} / L_{n}$. (b) The strong ETG turbulence with $\eta_{e}=5.0, \gamma \sim 0.20 v_{T e} / L_{n}$. 


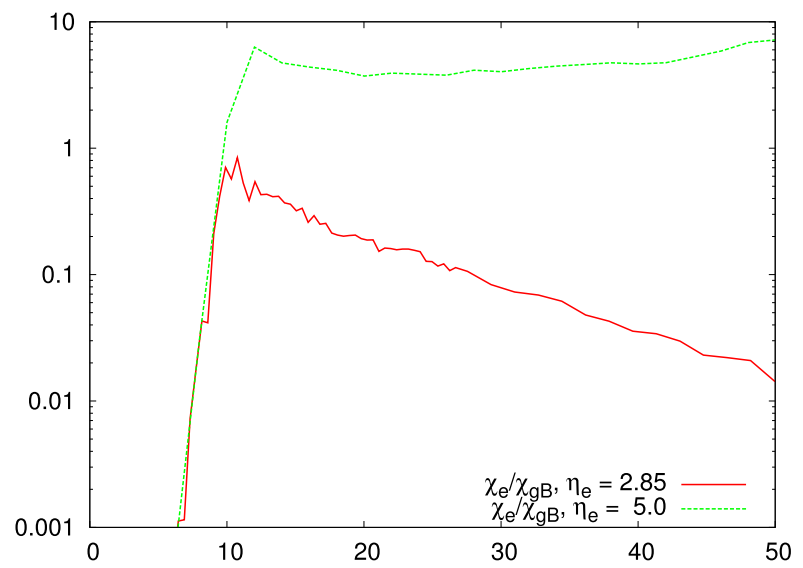

FIG. 8. Time histories of $\chi_{e} / \chi_{g B}$ for weak ETG turbulence with $\eta_{e}=2.85$ and the strong ETG turbulence with $\eta_{e}=5.0$. The time is normalized by the inverse of the linear growth rate $\gamma$ of the most unstable ETG mode at each $\eta_{e}$.

where $\Delta t=2.0 / \gamma$ is a time averaging window, $p_{0}^{\prime}$ is the radial derivative of the equilibrium pressure, and the electron heat flux $Q_{e}$ is defined as

$$
\begin{aligned}
Q_{e}= & \int \frac{c}{B_{0}} \hat{\boldsymbol{x}} \cdot(\boldsymbol{b} \times \nabla \phi)\left(\frac{1}{2} m_{e} v_{\|}^{2}+\mu B_{0}\right) \\
& \times F \delta[(\boldsymbol{R}+\boldsymbol{\rho})-\boldsymbol{x}] \mathrm{d}^{6} Z .
\end{aligned}
$$

The measured values are normalized by the gyro-Bohm coefficient $\chi_{G B}=\rho_{T e}^{2} v_{T e} / L_{n}$. Figure 8 shows the time development of $\chi_{e} / \chi_{G B}$ for the weak and strong ETG turbulence simulations shown in Fig. 7. The difference of $\chi_{e}$ between the weak and strong turbulence cases in the initial saturation phase $(t \gamma \sim 20)$ is $\sim 20$ times, while the mixing length estimate or the ratio of $\gamma$ is only $\sim 4$ times. In addition, in the quasi-steady phase, the difference expands, and the weak turbulence case is almost quenched. This qualitative difference in the turbulent transport is attributed to the selforganization. In order to see how these turbulent structures affect transport levels, the cross phase between $\phi$ and $\tilde{p}_{e}$, the perturbation part of the electron pressure, is compared in Fig. 9. Here, we use the definition given in Ref. 31, and time and space average is the same as that in Eq. (21). The result shows that the cross-phase is not so different between the weak and strong turbulence cases, and thus, the transport levels are affected mainly by the amplitudes of non-zonal component of $\phi$. Figures 10 and 11 show the 2D turbulent energy spectra. The thick lines show the Rhines scale $k_{c}$. The difference of the turbulent structures between these two cases, especially in the turbulent anisotropy, is attributed to the change of $k_{c}$. Although the linear wave dispersion is different between the electron drift wave in Fig. 1(a) and the ETG mode in Fig. 1(b), the structures of $k_{c}$ are qualitatively
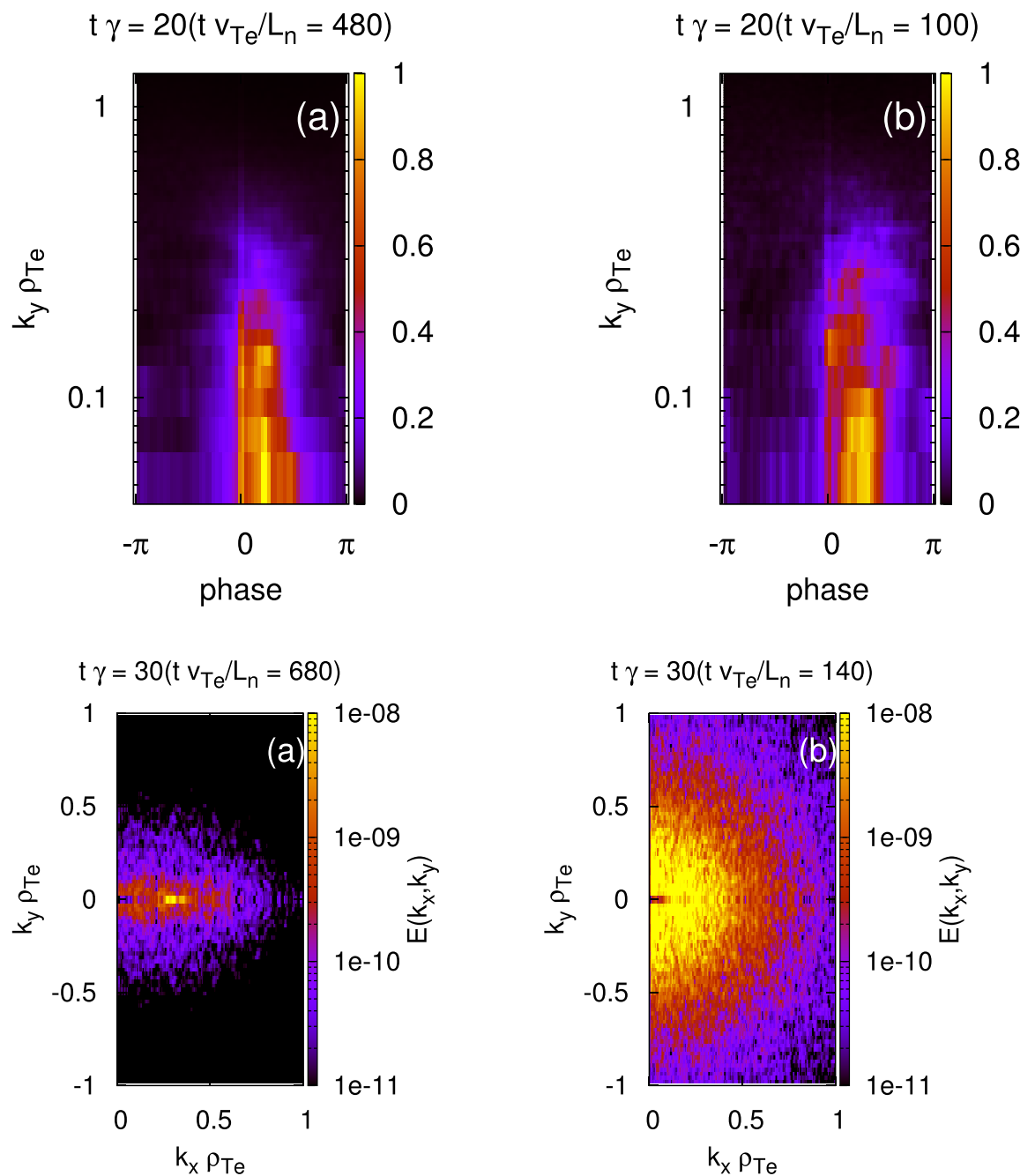

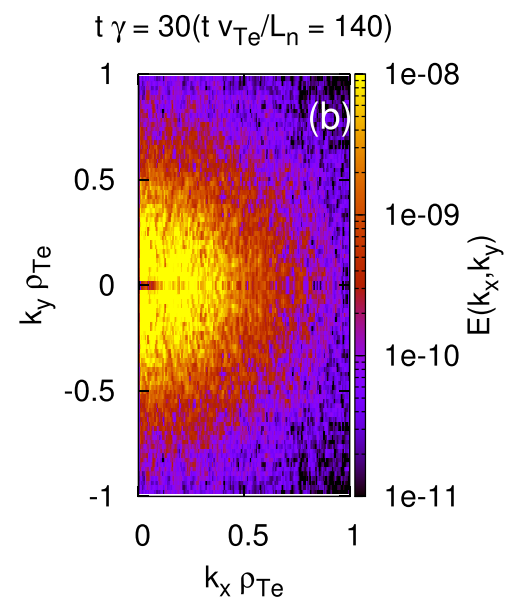

FIG. 9. The cross phase of $\phi$ and $\tilde{p}_{e}$ plotted at $t \gamma=20$ with (a) $\eta_{e}=2.85$ and (b) $\eta_{e}=5.0$.
FIG. 10. The 2D turbulent energy spec$\operatorname{tra} E_{k_{x}, k_{y}}$ observed in the weak and strong ETG turbulence with (a) $\eta_{e}=2.85$ and (b) $\eta_{e}=5.0$. The time average is taken with the window of $\Delta t=40 L_{n} / v_{T e}$ for (a) and $=10 L_{n} / v_{T e}$ for (b) in the quasisteady phases. 
similar. In the weak ETG turbulence in Fig. 11(a), $\omega_{r}$ is comparable to $\hat{\omega}_{t}$ at $k_{E} \rho_{T e} \sim 0.32$, and the turbulent cascade is impeded in the wave-like region with $k_{y}>k_{x}$. On the other hand, in the strong turbulence in Fig. 11(b), $\hat{\omega}_{t}$ exceeds $\omega_{r}$ around $k_{E} \rho_{T e} \sim 0.18$. The influence of the adiabatic ion response leads to the formation of isotropic turbulent structures.

\section{B. Turbulent energy spectra in ETG turbulence}

The self-organization picture in the HM model is based on the dual cascade, which shows the different power laws (7) in the lower and higher $k$ ranges. However, it is not trivial whether the dual cascade also exists in the GK model because the turbulent energy injection by the ETG mode and the energy dissipation by the Landau damping are distributed over wide spectral ranges. In order to clarify this point, we observe the turbulent energy spectrum $E_{k}$ and the energy injection rate $\Gamma_{k}$ in the shell averaged 1D wavenumber $k$ space. The latter is derived from the energy conservation of the GK model

$$
\begin{gathered}
\frac{d E_{k i n}}{d t}+\frac{d E_{f}}{d t}=\frac{d E_{c}}{d t}, \\
\frac{d E_{k i n}}{d t}=\frac{d}{d t} \int\left(\frac{1}{2} m_{e} v_{\|}^{2}+\mu B_{0}\right) F d^{6} Z, \\
\frac{d E_{f}}{d t}=\int q_{e}\langle\phi\rangle_{\alpha} \frac{\partial F}{\partial t} d^{6} Z \\
=\frac{d}{d t} \frac{1}{8 \pi \lambda_{D e}^{2}} \int\left[\rho_{s}^{2}|\hat{\nabla} \phi|^{2}+\tau|\phi|^{2}\right] d V, \\
\frac{d E_{c}}{d t}=\int q_{e}\langle\phi\rangle_{\alpha} C(F) d^{6} Z,
\end{gathered}
$$

where $\hat{\nabla} \equiv \rho_{T e} \nabla$. Since the field energy $E_{f}$ in the GK model corresponds to the turbulent energy $E$ in the HM model, the turbulent energy injection and dissipation are defined by transforming Eq. (25) into the $k$ space. $d E_{f} / d t$ is also defined as the energy transfer through the parallel streaming term, ${ }^{32}$ which can be written in the $k$ space as

$$
\begin{gathered}
\frac{d E_{f}}{d t}=\int q_{e} \frac{d \boldsymbol{R}}{d t} \cdot\langle\nabla \phi\rangle_{\alpha} F d^{6} Z=\sum_{k} \frac{d E_{k}}{d t}, \\
\frac{d E_{k}}{d t}=\int q_{e} v_{\|} k_{\|} \phi_{k} J_{0}\left(k \rho_{T e}\right) F_{k} d^{3} v .
\end{gathered}
$$

By using Eq. (28), the energy injection rate $\Gamma_{k}$ is defined as

$$
\Gamma_{k} \equiv \frac{1}{E_{k}} \frac{d E_{k}}{d t} .
$$

In the linear phase, the energy injection rate agrees with the linear growth rate at the most unstable $k$. Figures 12(a) and 12(b) show $E_{k}$ and $\Gamma_{k}$ in the weak and strong ETG turbulence simulations at the quasi-steady phase of $t \gamma=50$. In both cases, the most unstable wavenumber is given around $k \rho_{T e} \sim 0.4$, and the energy dissipation regions exist both in the higher and lower $k$ sides. However, the relative scales of energy injection, energy dissipation, and self-organization are different between these two cases. In the weak turbulence, $k_{c}$ shown in Fig. 11 is located in the relatively higher $k$ range, and the peak of zonal flows at $k_{Z F} \rho_{T e} \sim 0.30$ is rather close to the energy injection scale. Because of this scale overlap, the power law of the inverse energy cascade is not observed. On the other hand, in the strong turbulence, $k_{c}$ is shifted to lower $k$, and the energy containing scale develops up to $k_{E} \rho_{T e} \sim 0.05$, which is well separated from the energy injection scale. The resulting energy spectrum in Fig. 12(b) clearly shows the change of power laws between the lower and higher $k$ ranges, which support the existence of the dual cascade in the ETG turbulence. For these simulations, the adiabatic response scale $k_{\lambda} \rho_{T e} \sim 0.32$ is close to the most unstable wavenumber, and the inverse energy cascade with $E_{k} \propto k^{-11 / 3}$ is expected. However, the spectrum in Fig. 12(b) is rather close to $E_{k} \propto k^{-5 / 3}$. The discrepancy may be due to the remaining contribution from the Debye shielding term, which is still comparable to the adiabatic response term around $k \sim k_{\lambda}$.

\section{Impact of plasma parameter on self-organization}

In this section, we discuss the possibility of controlling the self-organization process by the nonlinear transfer rate $\hat{\omega}_{t}$. The weak and strong ETG turbulence simulations in Sec. III show that the anisotropy of the turbulent structures is changed depending on the competition of the Rhines scale and the adiabatic response scale. We investigate this effect more in detail in a systematic $\eta_{e}$ scan. It is noted that as shown in Fig. 1(b), $\eta_{e}$ mainly affects $\gamma$, while the change in $\omega_{r}$ is relatively small. Figures 13(a) and 13(b) show the time evolutions of the RMS turbulence velocity $U$ and the turbulent anisotropy $c_{y}$ in the $\eta_{e}$ scan. In the linear phase, eigenfunctions of the ETG mode
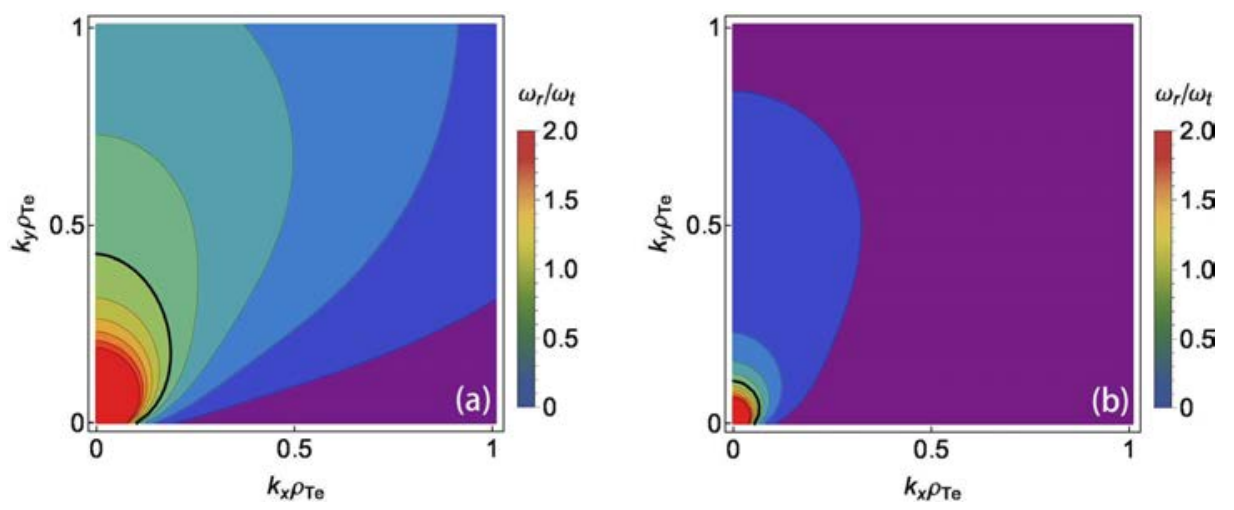

FIG. 11. The ratio of the real frequency of the ETG mode $\omega_{r}$ to the nonlinear transfer rate $\hat{\omega}_{t}$ is plotted on the $k_{x}-k_{y}$ plane. (a) and (b) The weak and strong ETG turbulence cases in Figs. 10(a) and 10(b). 

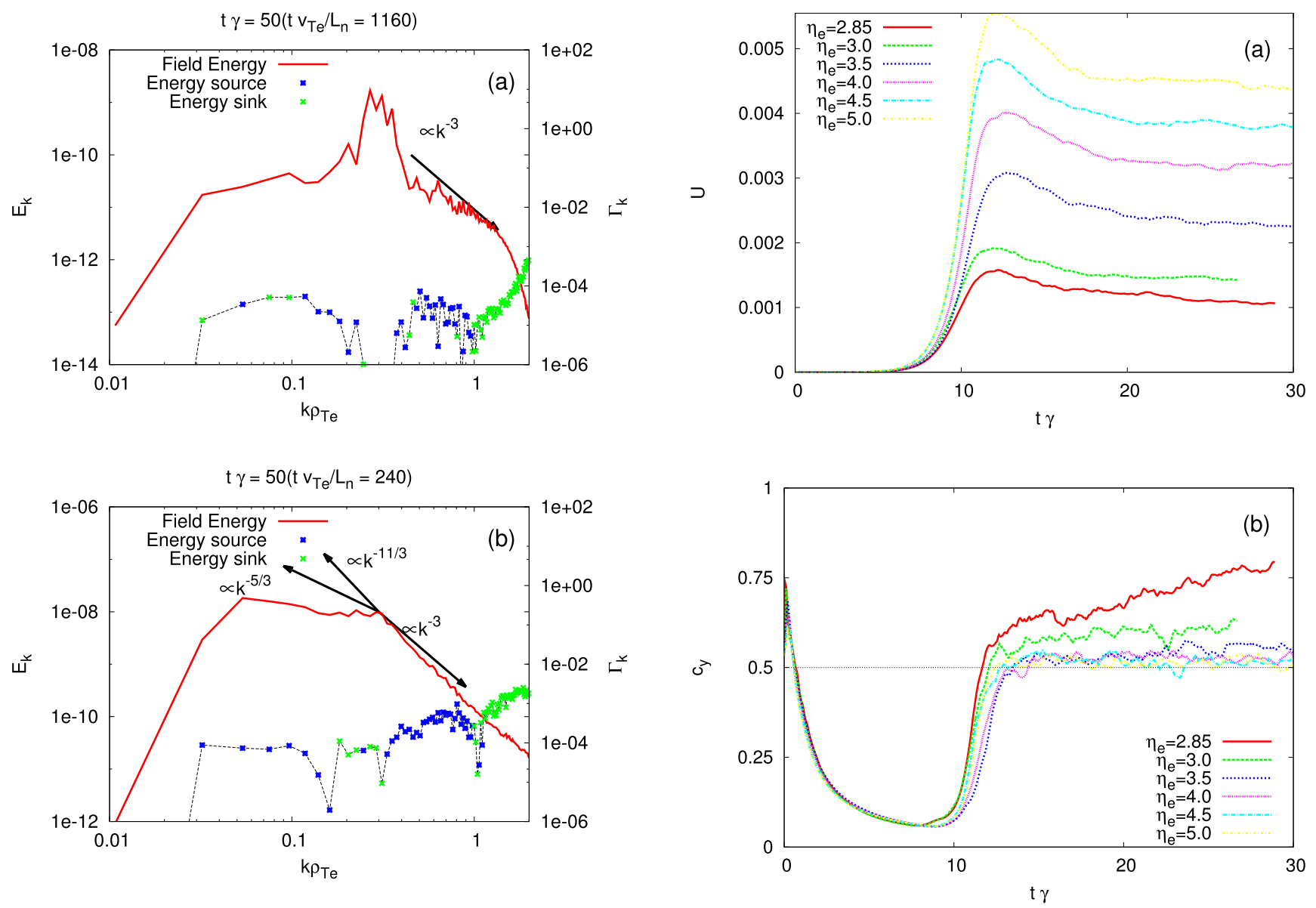

FIG. 12. The turbulent energy spectrum $E_{k}$ and the energy injection rate $\left|\Gamma_{k}\right|$ in the shell averaged 1D wavenumber $k$ space are shown for the ETG turbulence simulations with (a) $\eta_{e}=2.85$ and (b) $\eta_{e}=5.0$. The time average is taken at the quasi-steady phase $t \gamma=50$ with the window of $\Delta t \gamma \sim 2$. The energy injection rate is plotted by using its absolute value, and blue and green symbols indicate energy injection $(+)$ and dissipation $(\times)$, respectively. In (b), the power laws of the inverse energy cascade and the normal enstrophy cascade, (7) and (13), are shown by arrows.

produce streamers with $c_{y} \sim 0$, and in the early saturation phase, turbulent structures with $c_{y} \sim 0.5$ are produced due to the excitation of zonal modes. Although these features are common to all cases, in the quasi-steady phase, different turbulent structures are slowly formed depending on $\eta_{e}$. In Fig. 13(b), anisotropic turbulent structures emerge in the low $\eta_{e}$ cases with $\eta_{e} \leq 3.0$.

The parametric dependency of the turbulent anisotropy $c_{y}$ to the ratio of the linear mode frequency to the nonlinear transfer rate, $\omega_{r} / \hat{\omega}_{t}$, at the energy containing scale $k_{E}$ is shown in Fig. 14. Here, $\hat{\omega}_{t}(k)$ is given using the shell averaged spectrum of turbulence velocity $U(k)^{28}$

$$
\begin{gathered}
\epsilon(k)=\frac{1}{2} \int_{0.5 k}^{2 k} k^{2}\left|\phi_{k}\right|^{2} d k, \\
U(k)=\sqrt{2 \epsilon(k)} .
\end{gathered}
$$

The criterion parameter $k_{c} / k_{\lambda}$ is also plotted against $\omega_{r} / \hat{\omega}_{t}\left(k_{E}\right)$ in Fig. 14. In the figure, the weak and strong ETG turbulence simulations in Figs. 10(a) and 10(b) corresponds to $k_{c} / k_{\lambda} \sim 1.5$ and $\sim 0.64$, respectively. The result clearly shows that $c_{y}$ depends on $\omega_{r} / \hat{\omega}_{t}\left(k_{E}\right)$, and smaller $\eta_{e}$ cases

FIG. 13. The time evolutions of the RMS turbulent velocity $U$ and the turbulent anisotropy $c_{y}$ in the $\eta_{e}$ scan of ETG turbulence simulations with $k_{\|} / k_{y}=5.1 \times 10^{-4}$. The time is normalized by the inverse of the linear growth rate $\gamma$ of the most unstable ETG mode at each $\eta_{e}$.

with zonal flows give larger $\omega_{r} / \hat{\omega}_{t}\left(k_{E}\right)$. It is also shown that $\omega_{r} / \hat{\omega}_{t}\left(k_{E}\right)$ is almost proportional to $k_{c} / k_{\lambda}$, and zonal flows are generated for $k_{c} / k_{\lambda}>1$, as shown in decaying turbulence in Sec. III C. On the other hand, for $k_{c} / k_{\lambda}<1$, isotropic turbulence is formed. These qualitatively different selforganization processes are understood also in Fig. 15. While the strong ETG turbulence shows $k_{E}(t) \sim t^{-3 / 8}$, which suggests the inverse energy cascade hindered by the adiabatic

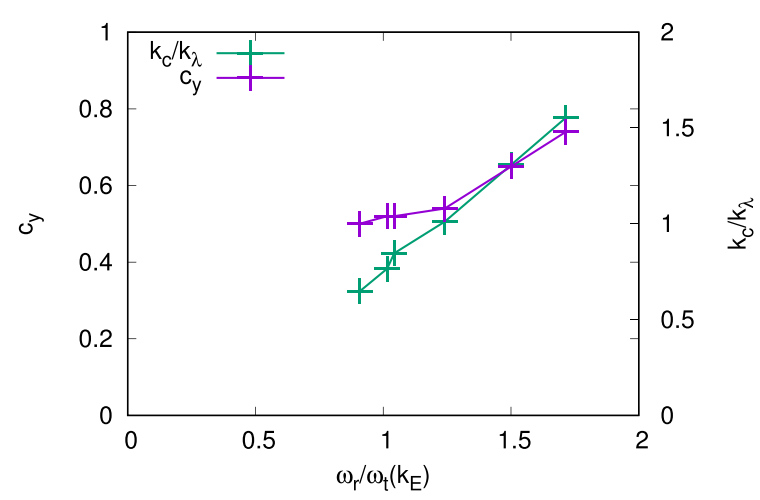

FIG. 14. The turbulent anisotropy $c_{y}$ is plotted against $\omega_{r} / \hat{\omega}_{t}\left(k_{E}\right)$, in which $k_{E}$ is measured at $t \gamma \sim 30$. In the $\eta_{e}$ scan in Fig. 13, anisotropic turbulent structure $c_{y}>0.5$ is formed for weak turbulence with $\omega_{r} / \hat{\omega}_{t}>1$. 


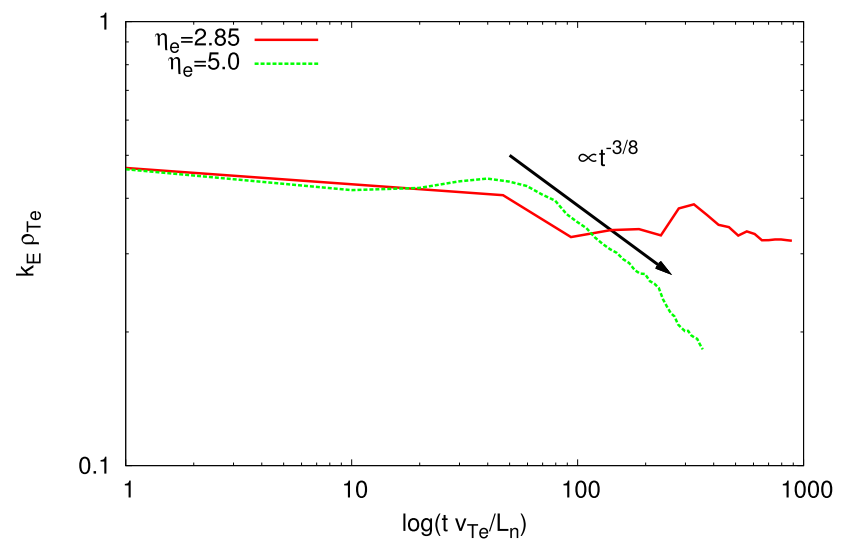

FIG. 15. The time development of the energy containing scale $k_{E}(t)$ is shown for the weak and strong ETG turbulence simulations (see Fig. 10). The inverse energy cascade for $k<k_{\lambda}$ is characterized by $k_{E}(t) \propto t^{-3 / 8}$.

response term, the weak ETG turbulence shows the condensation of turbulent spectra at the Rhines scale $k_{c}$.

\section{Modulational instability}

In this section, we consider the modulational instability ${ }^{15}$ as another candidate for the mechanism of zonal flow generation in the ETG turbulence. In fact, abrupt increase of the turbulent anisotropy $c_{y}$ (see Fig. 13(b)) is not by the inverse energy cascade, but by the direct excitation of zonal flows. This is clearly seen in the $2 \mathrm{D}$ energy spectra observed in the linear phase and the early saturation phase of the weak and the strong ETG turbulence simulations (see Fig. 16). The results indicate that zonal flows at $k_{Z F} \rho_{T e} \sim 0.30(0.35)$ are directly excited from linear streamers at $k_{y} \rho_{T e} \sim 0.35(0.45)$ for weak (strong) turbulence. This process is analyzed by calculating the modulational instability in the HM model, in which a zonal mode $\phi_{k_{q}, 0}$ is excited by its coupling with a pump wave $\phi_{0, k_{p}}$ and sideband modes $\phi_{k_{q},-k_{p}}$. Here, the pump wave is given at $k_{p} \rho_{T e}=0.35(0.45)$ based on the wavenumber of the ETG mode in Figs. 16(a) and 16(c). Figure 17 shows the $k_{q}$ dependency of the growth rate of the modulational instability. The result shows that the most unstable $k_{q}$ is slightly smaller than $k_{p}$, which is consistent with the above observation.

The coupling of these waves is directly measured by the bicoherence analysis, in which three-wave correlation is evaluated as

$$
b\left(\omega_{1}, \omega_{2}\right)=\frac{\left|\left\langle\phi\left(\omega_{1}\right) \phi\left(\omega_{2}\right) \phi^{*}\left(\omega_{1}+\omega_{2}\right)\right\rangle\right|^{2}}{\left\langle\left|\phi\left(\omega_{1}\right) \phi\left(\omega_{2}\right)\right|^{2}\right\rangle\left\langle\left|\phi^{*}\left(\omega_{1}+\omega_{2}\right)\right|^{2}\right\rangle},
$$
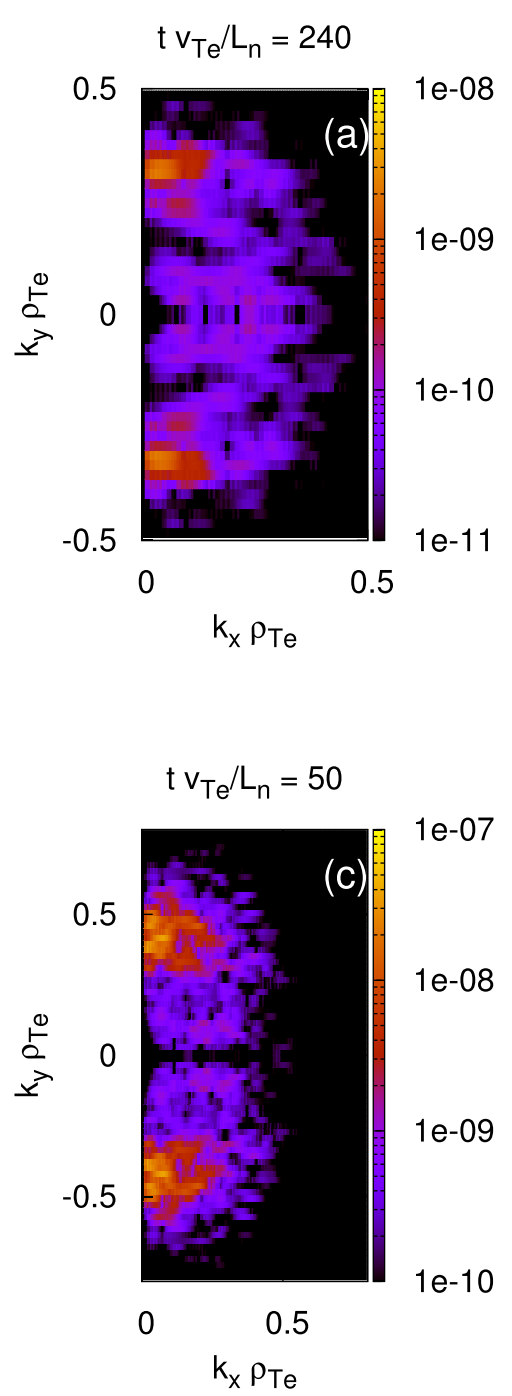
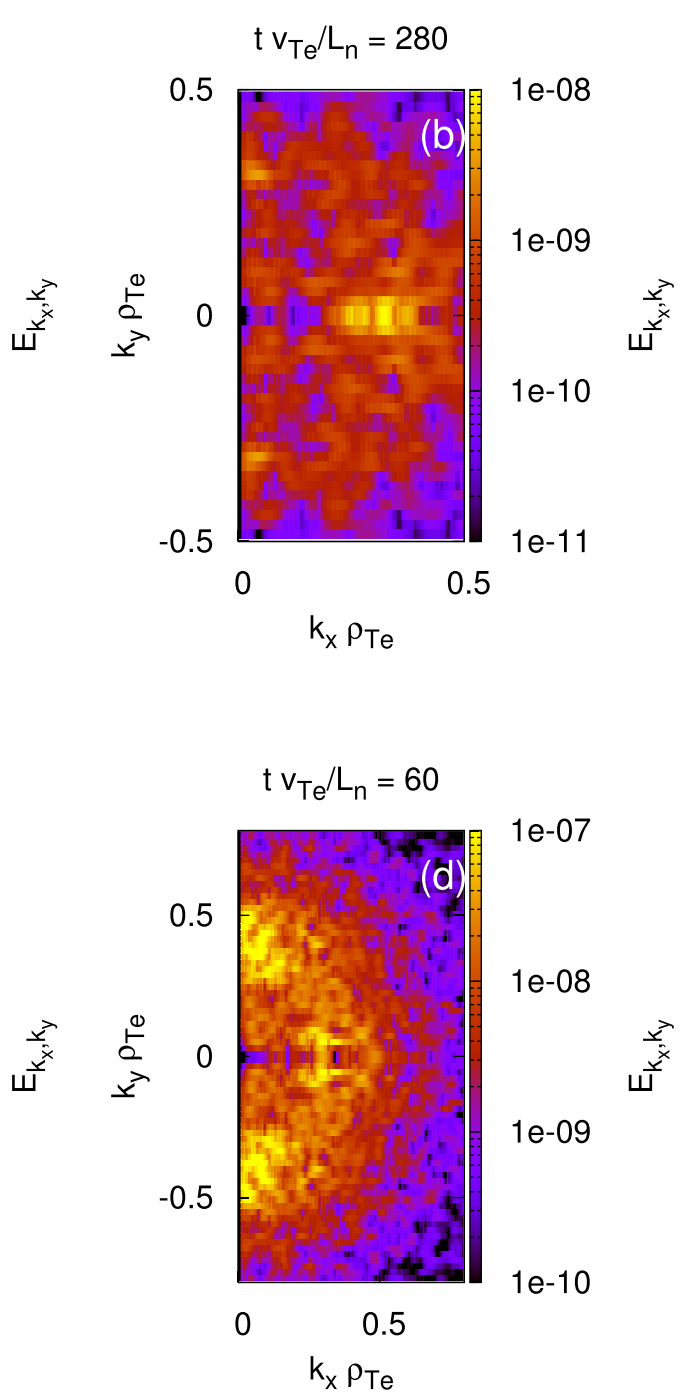

FIG. 16. The 2D energy spectra $E_{k_{k}}$ in (a), (c) linear phase and (b), (d) early saturation phase are shown for the weak (strong) ETG turbulence simulation with (a), (b) $\eta_{e}=2.85$, and (c), (d) $\eta_{e}=5.0$. 


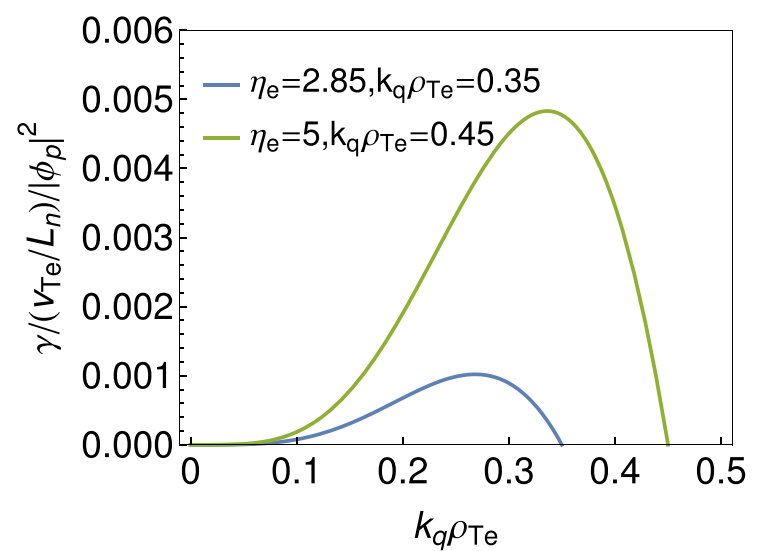

FIG. 17. The growth rate of the modulational instability is plotted against the wavenumber of the zonal mode $\left(k_{x}, k_{y}\right)=\left(k_{q}, 0\right)$. The wavenumber of the pump wave is chosen as $\left(k_{x}, k_{y}\right)=\left(0,0.35(0.45) \rho_{T e}^{-1}\right)$ based on Figs. 16(a) and 16(c).

where $\phi\left(\omega_{1}\right), \phi\left(\omega_{2}\right)$, and $\phi\left(\omega_{1}+\omega_{2}\right)$ are, respectively, frequency spectra of $\phi_{0, k_{p}}, \phi_{k_{q},-k_{p}}$, and $\phi_{k_{q}, 0}$, and $\langle\cdot\rangle$ denotes time average over the saturation phase. Figure 18 shows $b\left(\omega_{1}, \omega_{2}\right)$ for the saturation phase of the weak turbulence case for $t \gamma=12-14$. Here, the wavenumbers are chosen as $k_{p} \rho_{T_{e}}=0.35$ and $k_{q} \rho_{T_{e}}=0.28$ so that the coupling of the most unstable linear ETG mode and the most unstable zonal mode by the modulational instability is measured. In Fig. 18, strong coupling of the wave $\omega_{1}>0$ propagating in the direction of electron diamagnetic frequency to another wave $\omega_{2} \sim-\omega_{1}$ is observed. The resulting beat wave has the frequency $\omega_{1}+\omega_{2} \sim 0$, which is characteristic of the ZFs. The result suggests that the excitation of the ZFs at the saturation phase may be caused by the modulational instability of the linear ETG modes. This analysis cannot be applied to the strong turbulence case, because the saturation process is too fast to keep a sufficient time window for the bicoherence analysis. However, it can still be inferred from the ETG modes at $k_{x} \sim 0$ and ZFs at $k_{y} \sim 0$ in Figs. 16(c) and 16(d) that the same kind of modulational instability may exist. This result indicates that the self-organization process plays an essential role in dictating the final turbulent structure,

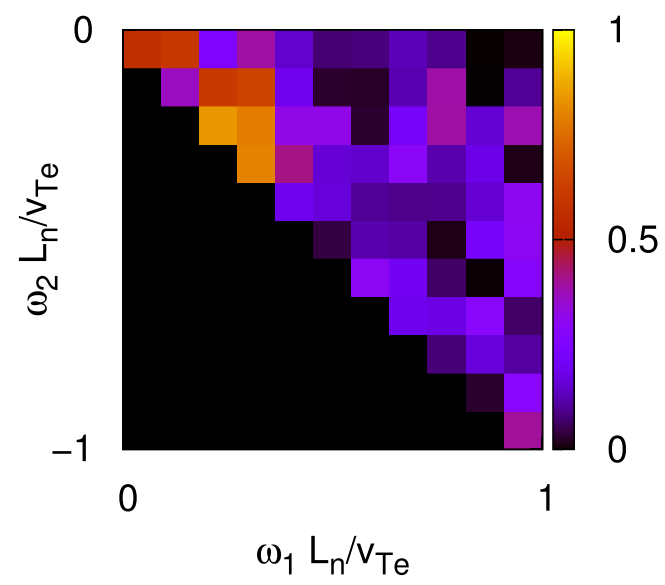

FIG. 18. The bicoherence $b\left(\omega_{1}, \omega_{2}\right)$ in a early saturation phase is shown for the weak ETG turbulence simulation at $t \gamma=12$. while transient mode coupling may be described by the modulational instability.

\section{SUMMARY AND DISCUSSION}

In this work, we have discussed the impacts of the plasma parameters on the self-organization of the electron turbulence based on the HM model, which is derived by taking the 2D fluid limit of the GK model. In the HM model, the selforganized turbulent structures are generated at the Rhines scale $k_{c}$, at which the linear mode frequency $\omega_{r}$ and the nonlinear transfer rate $\omega_{t}$ become comparable, $\omega_{r}\left(k_{c}\right) \sim \omega_{t}\left(k_{c}\right)$. As the short wavelength approximation is not satisfied in the ETG turbulence due to the typical scale $k \rho_{T e} \lesssim 1$, the role of the adiabatic response term should be considered. In order to see this effect, the impact of the criterion parameter $k_{c} / k_{\lambda}$ on turbulent structures is investigated, where $k_{\lambda}$ is defined at which the adiabatic response term becomes comparable to the Debye shielding term. The scale and the anisotropy of the self-organized turbulent structure are controlled by varying this parameter through the plasma parameters such as the density and temperature gradient.

In the decaying turbulence, in which the initial perturbations at given wavenumber are relaxed through the turbulent cascade, the applicability of the self-organization picture in the HM model is confirmed by showing the similarity of the zonal flow generation between the HM and GK models. We then demonstrated that the anisotropy of the turbulence structure is changed by controlling the initial amplitude of perturbation, which dictates $k_{c} / k_{\lambda}$. It is found that the weak and strong turbulence cases with $k_{c} / k_{\lambda}>1$ and $k_{c} / k_{\lambda}<1$ lead to zonal flows and isotropic turbulent structures, respectively, and in the strong turbulence case, the power law of $k_{E}(t)$ suggests that the inverse energy cascade is retarded by the adiabatic response term.

The self-organization process is investigated more in detail in the ETG turbulence, in which the scales of energy injection, energy dissipation, and self-organization are selfconsistently determined. The turbulent energy spectrum of the strong turbulence case clearly shows the power laws of the dual cascades, the inverse energy cascade, and the normal enstrophy cascade in the lower and higher $k$ ranges. The controllability of turbulent structures is examined by systematic $\eta_{e}$ scan and it is shown that a turbulent anisotropy is changed depending on the turbulence intensity. The plot of the ratio of the linear mode frequency to the nonlinear turbulent transfer rate at the energy containing scale $\omega_{r} / \omega_{t}\left(k_{E}\right)$ shows that the mechanism that determines the anisotropy of the turbulence structure is relevant also in the ETG turbulence.

In the future work, the self-organization process will be investigated by taking account of missing physical effects such as magnetic shear and toroidal mode coupling.

\section{ACKNOWLEDGMENTS}

This work was carried out using the HELIOS supercomputer system at Computational Simulation Center of International Fusion Energy Research Center (IFERC-CSC), Aomori, Japan, under the Broader Approach collaboration 
between Euratom and Japan, implemented by Fusion for Energy and JAEA, "Plasma Simulator" (FUJITSU FX100) of NIFS, and "BX900" of JAEA. One of the authors (C.K.) is supported by the JAEA research fellowship.

${ }^{1}$ W. Horton, Rev. Mod. Phys. 71, 735 (1999).

${ }^{2}$ T. Görler and F. Jenko, Phys. Rev. Lett. 100, 185002 (2008).

${ }^{3}$ S. Maeyama, Y. Idomura, T.-H. Watanabe, M. Nakata, M. Yagi, N. Miyato, A. Ishizawa, and M. Nunami, Phys. Rev. Lett. 114, 255002 (2015).

${ }^{4}$ N. Howard, C. Holland, A. White, M. Greenwald, and J. Candy, Nucl. Fusion 56, 014004 (2016).

${ }^{5}$ B. W. Stallard, C. M. Greenfield, G. M. Staebler, C. L. Rettig, M. S. Chu, M. E. Austin, D. R. Baker, L. R. Baylor, K. H. Burrell, J. C. DeBoo, J. S. DeGrassie, E. J. Doyle, J. Lohr, G. R. McKee, R. L. Miller, W. A. Peebles, C. C. Petty, R. I. Pinsker, B. W. Rice, T. L. Rhodes, R. E. Waltz, and L. Zeng, Phys. Plasmas 6, 1978 (1999).

${ }^{6}$ H. Shirai, M. Kikuchi, T. Takizuka, T. Fujita, Y. Koide, G. Rewoldt, D. Mikkelsen, R. Budny, W. M. Tang, Y. Kishimoto, Y. Kamada, T. Oikawa, O. Naito, T. Fukuda, N. Isei, Y. Kawano, and M. Azumi, Nucl. Fusion 39, 1713 (1999)

${ }^{7}$ D. R. Smith, S. M. Kaye, W. Lee, E. Mazzucato, H. K. Park, R. E. Bell, C. W. Domier, B. P. Leblanc, F. M. Levinton, N. C. Luhmann, J. E. Menard, and H. Yuh, Phys. Rev. Lett. 102, 225005 (2009).

${ }^{8}$ W. Dorland, F. Jenko, M. Kotschenreuther, and B. N. Rogers, Phys. Rev. Lett. 85, 5579 (2000).

${ }^{9}$ F. Jenko, W. Dorland, M. Kotschenreuther, and B. N. Rogers, Phys. Plasmas 7, 1904 (2000).

${ }^{10}$ Y. Idomura, S. Tokuda, and Y. Kishimoto, Nucl. Fusion 45, 1571 (2005).
${ }^{11}$ Y. Idomura, M. Wakatani, and S. Tokuda, Phys. Plasmas 7, 3551 (2000).

${ }^{12} \mathrm{~J}$. Li and Y. Kishimoto, Phys. Plasmas 11, 1493 (2004).

${ }^{13}$ G. J. Colyer, A. A. Schekochihin, F. I. Parra, C. M. Roach, M. A. Barnes, Y.-C. Ghim, and W. Dorland, Plasma Phys. Control. Fusion 59, 55002 (2017).

${ }^{14}$ L. Chen, Z. Lin, and R. White, Phys. Plasmas 7, 3129 (2000).

${ }^{15} \mathrm{~J}$. Li and Y. Kishimoto, Phys. Plasmas 9, 1241 (2002).

${ }^{16}$ F. Jenko and W. Dorland, Phys. Rev. Lett. 89, 225001 (2002).

${ }^{17}$ Y. Idomura, Phys. Plasmas 13, 080701 (2006).

${ }^{18}$ A. Hasegawa and K. Mima, Phys. Rev. Lett. 39, 205 (1977).

${ }^{19}$ J. G. Charney, J. Meteorol. 4, 135 (1947).

${ }^{20}$ A. A. Schekochihin, S. C. Cowley, W. Dorland, G. W. Hammett, G. G. Howes, G. G. Plunk, E. Quataert, and T. Tatsuno, Plasma Phys. Controlled Fusion 50, 124024 (2008).

${ }^{21}$ T. Tatsuno, W. Dorland, A. Schekochihin, G. Plunk, M. Barnes, S. C. Cowley, and G. Howes, Phys. Rev. Lett. 103, 015003 (2009).

${ }^{22}$ A. B. Navarro, P. Morel, M. Albrecht-Marc, D. Carati, F. Merz, T. Görler, and F. Jenko, Phys. Rev. Lett. 106, 055001 (2011).

${ }^{23}$ Y. Idomura, M. Ida, S. Tokuda, and L. Villlard, J. Comput. Phys. 226, 244 (2007).

${ }^{24}$ X. Q. Xu and M. N. Rosenbluth, Phys. Fluids B 3, 627 (1991).

${ }^{25}$ Y. Idomura, H. Urano, N. Aiba, and S. Tokuda, Nucl. Fusion 49, 065029 (2009).

${ }^{26}$ R. H. Kraichnan, Phys. Fluids 10, 1417 (1967).

${ }^{27}$ G. K. Vallis and M. E. Maltrud, J. Phys. Oceanogr. 23, 1346 (1993).

${ }^{28}$ P. B. Rhines, J. Fluid Mech. 69, 417 (1975).

${ }^{29}$ T. Watanabe, H. Fujisaka, and T. Iwayama, Phys. Rev. E 55, 5575 (1997).

${ }^{30}$ Y. Idomura, S. Tokuda, and M. Wakatani, Phys. Plasmas 6, 4658 (1999).

${ }^{31}$ T. Dannert and F. Jenko, Phys. Plasmas 12, 072309 (2005).

${ }^{32}$ R. Hatzky, T. M. Tran, A. Könies, R. Kleiber, and S. J. Allfrey, Phys. Plasmas 9, 898 (2002). 